\title{
Gene copy number and function of the APL1 immune factor changed during Anopheles evolution
}

\author{
Christian Mitri ${ }^{1,2}$, Emmanuel Bischoff ${ }^{1,2}$, Karin Eiglmeier ${ }^{1,2}$, Inge Holm ${ }^{1,2}$, Constentin Dieme ${ }^{1,2,3}$, \\ Emma Brito-Fravallo ${ }^{1,2}$, Abbasali Raz ${ }^{4}$, Sedigheh Zakeri ${ }^{4}$, Mahdokht I. K. Nejad ${ }^{4}$, Navid D. Djadid ${ }^{4}$, \\ Kenneth D. Vernick ${ }^{1,2^{*}}$ and Michelle M. Riehle ${ }^{5^{*}}$
}

\begin{abstract}
Background: The recent reference genome assembly and annotation of the Asian malaria vector Anopheles stephensi detected only one gene encoding the leucine-rich repeat immune factor APL1, while in the Anopheles gambiae and sibling Anopheles coluzzii, APL1 factors are encoded by a family of three paralogs. The phylogeny and biological function of the unique APL1 gene in An. stephensi have not yet been specifically examined.

Methods: The APL1 locus was manually annotated to confirm the computationally predicted single APL1 gene in An. stephensi. APL1 evolution within Anopheles was explored by phylogenomic analysis. The single or paralogous APL1 genes were silenced in An. stephensi and An. coluzzii, respectively, followed by mosquito survival analysis, experimental infection with Plasmodium and expression analysis.

Results: APL1 is present as a single ancestral gene in most Anopheles including An. stephensi but has expanded to three paralogs in an African lineage that includes only the Anopheles gambiae species complex and Anopheles christyi. Silencing of the unique APL1 copy in An. stephensi results in significant mosquito mortality. Elevated mortality of APL1-depleted An. stephensi is rescued by antibiotic treatment, suggesting that pathology due to bacteria is the cause of mortality, and indicating that the unique APL1 gene is essential for host survival. Successful Plasmodium development in An. stephensi depends upon APL1 activity for protection from high host mortality due to bacteria. In contrast, silencing of all three APL1 paralogs in An. coluzzii does not result in elevated mortality, either with or without Plasmodium infection. Expression of the single An. stephensi APL1 gene is regulated by both the Imd and Toll immune pathways, while the two signaling pathways regulate different APL1 paralogs in the expanded APL1 locus.

Conclusions: APL1 underwent loss and gain of functions concomitant with expansion from a single ancestral gene to three paralogs in one lineage of African Anopheles. We infer that activity of the unique APL1 gene promotes longevity in An. stephensi by conferring protection from or tolerance to an effect of bacterial pathology. The evolution of an expanded APL1 gene family could be a factor contributing to the exceptional levels of malaria transmission mediated by human-feeding members of the An. gambiae species complex in Africa.
\end{abstract}

Keywords: Mosquito, Insect immunity, Gene family, Gene essentiality, Gene neofunctionalization

\footnotetext{
*Correspondence: kenneth.vernick@pasteur.fr; mriehle@mcw.edu

1 Unit of Insect Vector Genetics and Genomics, Department of Parasites and Insect Vectors, Institut Pasteur, Paris, France

${ }^{5}$ Department of Microbiology and Immunology, Medical College of Wisconsin, Milwaukee, WI, USA

Full list of author information is available at the end of the article
}

\section{Background}

Malaria remains a serious global public health concern. Human malaria is transmitted by Anopheles mosquitoes and among > 450 extant Anopheles species,

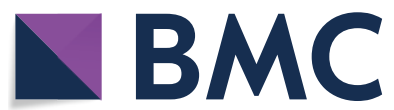

(c) The Author(s) 2020. This article is licensed under a Creative Commons Attribution 4.0 International License, which permits use, sharing, adaptation, distribution and reproduction in any medium or format, as long as you give appropriate credit to the original author(s) and the source, provide a link to the Creative Commons licence, and indicate if changes were made. The images or other third party material in this article are included in the article's Creative Commons licence, unless indicated otherwise in a credit line to the material. If material is not included in the article's Creative Commons licence and your intended use is not permitted by statutory regulation or exceeds the permitted use, you will need to obtain permission directly from the copyright holder. To view a copy of this licence, visit http://creativeco mmons.org/licenses/by/4.0/. The Creative Commons Public Domain Dedication waiver (http://creativecommons.org/publicdomain/ zero/1.0/) applies to the data made available in this article, unless otherwise stated in a credit line to the data. 
approximately 40 are considered dominant malaria vector species (DVS) [1]. About 90\% of global Plasmodium falciparum transmission occurs in Africa, where the most important DVS on earth are members of the Anopheles gambiae species complex (hereafter, Gambiae complex), including the widespread Anopheles coluzzii. An important Asian DVS is Anopheles stephensi, which has recently been recognized as an invasive vector species, expanding disease transmission along with its geographical range [2, 3].

The heterogeneity among Anopheles species for malaria vectorial capacity can have multiple causes. The first is host-feeding behavior, because animal-feeding species do not have the opportunity to acquire and transmit a human pathogen. Consequently, human-biting preference is the most fundamental prerequisite of malaria vectorial capacity $[4,5]$. Among human-feeding DVS, there is apparent variation in vectorial capacity, suggested by large geographical differences in human malaria infection prevalence, with about $90 \%$ of global prevalence located in Africa [6]. Some of this global geographical variation could be caused by ecology, if some niches, for example in humid sub-Saharan Africa, are particularly favorable to mosquito abundance and longevity, promoting malaria transmission [7-9]. Finally, vector genetic differences can also underlie physiological difference in vector competence for $P$. falciparum in nature [10-12], but the mechanisms underlying Anopheles susceptibility to human malaria in nature are not understood. Several tens of Anopheles genes are known from laboratory studies to control malaria infection of the vector, but involvement of these genes in modulating natural transmission has not been confirmed by genetic association in the natural vector population.

The best described mechanism of mosquito immunity in laboratory studies is a ternary immune complex in the Gambiae complex, comprised of the leucine-rich repeat (LRR) proteins APL1 and LRIM1 and the complementlike factor TEP1 [13-15]. APL1 is present in the Gambiae complex as a family of three paralogs, APL1A, APL1B and APL1C [14]. The paralogs display distinct spectra of protection for different pathogen classes [16-18]. APL1A activity inhibits development of the human parasite $P$. falciparum, while APL1C activity inhibits rodent malaria species [14] and APL1B modulates protection against both P. falciparum and the rodent parasites [17].

The recent reference genome assembly and annotation of the Asian malaria vector An. stephensi revealed only one APL1 gene rather than three paralogs as in the Gambiae complex [19]. Here, we experimentally validate the computationally predicted single APL1 gene in $A n$. stephensi. Phylogenomic analysis indicates that a single copy of APL1 represents the ancestral anopheline state, while the expansion to three APL1 paralogs is derived, and among DVS is found only in the African lineage that includes the Gambiae complex. Anopheles stephensi APL1 was previously tested for effect on P. falciparum [20] and response to kinase signaling [21], but the biological function of the unique APL1 gene has not yet been specifically examined, nor compared to the function of the expanded APL1 locus. We find that the single-copy ancestral APL1 gene and the expanded APL1 locus display distinct functional phenotypes for host survival and protection against Plasmodium infection. The expanded APL1 locus is found in the most efficient DVS in the world, the Gambiae complex, which poses the question whether the apparent correlation of APL1 copy number with efficient malaria transmission is accidental or, at least in part, causal.

\section{Methods}

\section{Mosquitoes}

Anopheles stephensi SDA-500 strain was initiated in Pakistan [22] and Anopheles coluzzii Ngousso strain was initiated in Cameroon [23]. Both strains are housed in the insectaries of the CEPIA platform at the Institut Pasteur, Paris. Mosquitoes were reared under standard conditions at $26^{\circ} \mathrm{C}$ and $80 \%$ relative humidity, with a $12 \mathrm{~h}$ light/dark cycle and continuous access to $10 \%$ sucrose solution in cotton pads [17].

Anopheles stephensi samples used for APL1 population variation analysis were 6 individuals from a colony initiated at Chabahar, Iran in 2011, 6 individuals from a colony initiated at Bandar-Abbas, Iran in 2008 (both strains maintained at the Institut Pasteur of Iran) and 1 wild-caught individual from Bandar-Abbas. An 800 bp portion of the APL1 coding sequence was amplified from individuals using An. stephensi APL1 primers Iran40F and Iran06R. Amplicons of individuals were sequenced, and variant calls were confirmed on both strands by visual examination of $\mathrm{ABI}$ sequence chromatogram trace files. Anopheles coluzzii APL1 sequences were previously published, generated from the Ngousso colony [24] or wild population [25] and deposited in public archives.

\section{Phylogenetic analysis of Anopheles APL1 gene copy number}

The APL1 locus was manually sequenced and sized by PCR in the Anopheles stephensi SDA-500 colony housed at Institut Pasteur. Strategy and primers used are indicated in Additional file 1: Figure S1. The annotated An. stephensi APL1 genes in the VectorBase genome database [26] are ASTE016290 in the An. stephensi SDA-500 strain and ASTEI02571 in the An. stephensi Indian strain. The VectorBase assemblies and annotations used, current as of January 2019 were: SDA-500 strain, assembly AsteS1, 
gene set: AsteS1.7, dated 22 October 2018; and Indian strain, assembly AsteI2, gene set AsteI2.3, dated 21 February 2017.

For phylogenetic analysis of APL1 copy number as presented in Additional file 2: Figure S2, APL1 orthologues for all Anopheles species genome assemblies were obtained from VectorBase and sequence was extracted for a window of 60,000 base pairs (bp) centered on the APL1 orthologue(s). Sequences were compared and visualized in a pair-wise fashion using the tBlastX algorithm within the Double Act interface of the Artemis Comparison Tool [27] and visualized using Easyfig [28] to illustrate the number of APL1 family genes across species. Forward and reverse matches were colored the same and percent ID cut-offs were set to a minimum of $50 \%$ (light pink in Additional file 2: Figure S2 represents a 50\% match and bright red $100 \%$ match, areas with less than $50 \%$ match are not depicted in color). Each mosquito species was compared directly to the An. gambiae PEST genome, the most mature Anopheles genome in which the APL1 gene family was originally annotated [14].

For structural comparison of An. stephensi APL1 with An. gambiae APL1C, peptide sequences were obtained from VectorBase An. stephensi assembly SDA-500 and An. gambiae assembly AgamP4. Protein motif predictions were carried out and compared using InterPro [29].

\section{Gene silencing}

Double-stranded RNA (dsRNA) specific for target genes was synthesized using the T7 Megascript Kit (Ambion, Waltham MA, USA) as described [16] using indicated primers (Additional file 3: Table S1). For each targeted gene, $500 \mathrm{ng}$ of dsRNA (but not more than $207 \mathrm{nl}$ volume, depending on concentration) was injected into the thorax of cold-anesthetized 1-day-old female mosquitoes using a Nanoject II Auto-Nanoliter Injector (Drummond Scientific, Broomall PA, USA). Mosquitoes were injected with dsRNA specific for the target gene, or with the control dsRNA, dsGFP. The efficiency of gene silencing was monitored 4 days after dsRNA injection in pools of 8 mosquitoes as follows. After total RNA extraction, cDNA synthesis was performed using M-MLV reverse transcriptase and random hexamers (Invitrogen, Carlsbad CA, USA). For each sample, $1 \mu \mathrm{g}$ of total RNA was used in each of three independent cDNA synthesis reactions. Triplicates were pooled and used as template for $\mathrm{qPCR}$ analysis. Real-time PCR was performed using an ABI Prism 7900HT sequence detector (Applied Biosystems, Foster City CA, USA). Reactions were prepared in a total volume of $20 \mu \mathrm{l}$ using SYBR Green PCR master mix (Applied Biosystems, Foster City CA, USA) and $900 \mathrm{nM}$ primers with three serial dilutions of cDNA, each dilution assayed in triplicate. Primers used for verification of gene silencing are indicated (Additional file 3: Table S1). PCR conditions were $95{ }^{\circ} \mathrm{C}$ for $10 \mathrm{~min}$ followed by 40 cycles of $95^{\circ} \mathrm{C}$ for $15 \mathrm{~s}, 55^{\circ} \mathrm{C}$ for $15 \mathrm{~s}$ and $60{ }^{\circ} \mathrm{C}$ for $45 \mathrm{~s}$. mRNA level was normalized to self (An. stephensi or An. coluzzii) ribosomal protein rpS7 mRNA in each sample and each gene silencing condition was compared to the control treated with dsGFP.

\section{Plasmodium infection and phenotyping}

Mosquitoes were fed on mice infected with Plasmodium yoelii strain delta-p230p-GFP [30] at 8-12\% parasitemia with mature gametocytes. For parasite development, mosquitoes were maintained at $24{ }^{\circ} \mathrm{C}$ and $70 \%$ relative humidity on $10 \%$ sucrose or $10 \%$ sucrose supplemented with penicillin $62.5 \mu \mathrm{g} / \mathrm{ml}$, streptomycin $100 \mu \mathrm{g} / \mathrm{ml}$ and gentamicin $50 \mu \mathrm{g} / \mathrm{ml}$. To measure $P$. yoelii infection, mosquito midguts were dissected at day 8 post-infection and oocysts were counted by fluorescence microscopy. Infection phenotypes measured were oocyst infection prevalence, which is the proportion of mosquitoes carrying $\geq$ 1 oocyst among the total number of dissected mosquitos and oocyst intensity, which is the oocyst count in mosquitoes with $\geq 1$ oocyst. Mosquito infection phenotypes were determined for at least two independent biological replicates of $\geq 30$ dissected mosquitoes per replicate.

Differences in infection prevalence were statistically tested using the Chi-square test and analysis of oocyst intensity differences used the Wilcoxon signed rank nonparametric test. Statistical differences in prevalence and intensity were first tested independently for each replicate as described above and $P$-values were empirically determined using 100,000 Monte-Carlo permutations. Following independent statistical tests for each replicate and when the direction of change of each independent replicate was concordant, the $P$-values from independent tests of significance were statistically combined using the meta-analytical approach of Fisher [31]. All statistical analyses were carried out using $\mathrm{R}$ [32].

\section{Mosquito mortality curves}

Mosquito mortality was monitored in cages of at least 50 mosquitoes, recorded every 2 days until all mosquitoes died. Treatment with dsRNA was performed in 3-dayold mosquitoes and recording of mortality began 4 days after dsRNA injection in 7-day-old mosquitoes. Bloodfeeding with or without $P$. yoelii was done 4 days after dsRNA injection in 7-day-old mosquitoes and recording of mortality began 3 days following the normal or infected blood meal in 10-day-old mosquitoes. Beginning at adult emergence, mosquitoes were maintained with $10 \%$ sucrose and in the case of antibiotic treatment, supplemented with penicillin $62.5 \mu \mathrm{g} / \mathrm{ml}$, streptomycin 100 $\mu \mathrm{g} / \mathrm{ml}$ and gentamicin $50 \mu \mathrm{g} / \mathrm{ml}$. Two to three replicates 
were performed for each condition tested. A Cox proportional hazards regression model was fitted to the data using treatments as predictor terms $[33,34]$.

\section{Results}

Phylogeny of APL1 gene expansion from a unique ancestor Recent in silico annotation of the An. stephensi reference genome detected a single APL1 gene [19]. This is in contrast to the Gambiae species complex, where APL1 is comprised of a family of three paralogs, APL1A, APL1B and APL1C, with distinct roles in immunity [14, 16]. Because assembly of short-read sequences can be problematic for paralogous families, we first wished to confirm the in silico single-gene model for An. stephensi APL1. The An. stephensi SDA-500 assembly contains an unresolved region with unjoined contigs that could potentially conceal the existence of other APL1 paralogs between APL1 (ASTE016290) and the adjacent gene ASTE008334. We manually sized and sequenced $\sim 7 \mathrm{~kb}$ of the APL1 locus in SDA-500, which closed the sequence assembly gaps and confirmed the presence of a single APL1 gene in An. stephensi SDA-500 (Additional file 1: Figure S1, Additional file 4: Alignment S1).

We then examined the phylogeny of APL1 in all 19 public reference genomes from 18 Anopheles species, which includes two independent assemblies for An. stephensi $[19,35]$. We accepted genome annotations and did not manually verify the structure of APL1 orthologs in the other genome assemblies as we did above for An. stephensi and was previously done for An. gambiae-An. coluzzii [14], because we only performed further functional experiments with the latter two species. A single APL1 gene was identified in 12 species, including An. stephensi, while the genome assemblies that include the Gambiae complex and An. christyi display an expanded APL1 gene family (Fig. 1, Additional file 2: Figure S2). The Gambiae complex members each carry three APL1 paralogs, with the same locus structure as previously described for the sister taxa An. gambiae and An. coluzzii [14, 25]. The African species $A n$. christyi, the closest sequenced relative outside the Gambiae complex, contains at least two APL1 genes and likely a third, but resolution is limited because the $A n$.

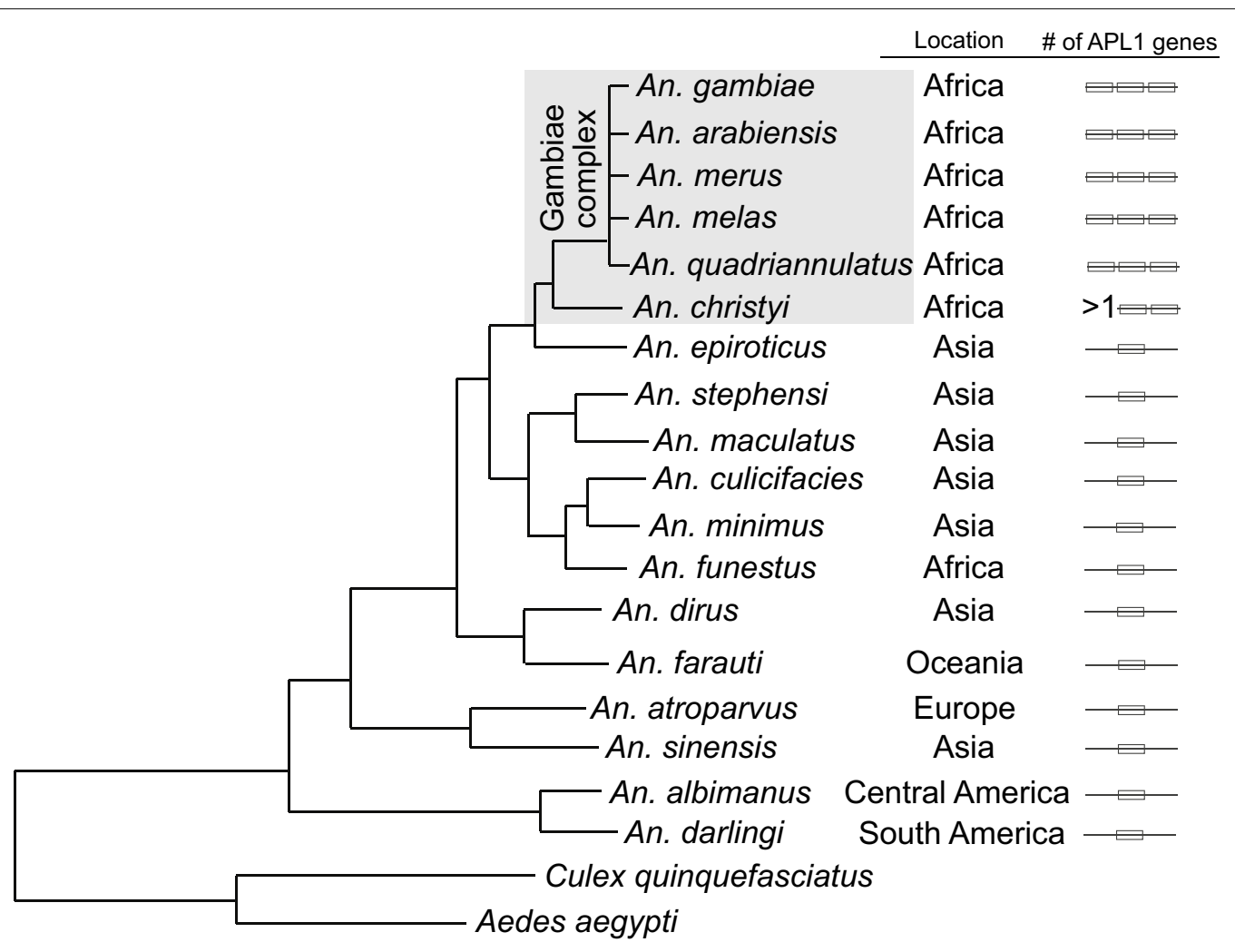

Fig. 1 The APL1 gene underwent an expansion in an African Anopheles lineage. Anopheles phylogenetic tree indicates the number of APL1 gene paralogs present in the genome of 18 Anopheles species. Geographical locations of species and the number of APL1 genes in each species are indicated in columns, "Location" and "\# of APL1 genes", respectively. Anopheles species worldwide, including An. funestus in Africa, carry a single APL1 gene, which is the ancestral state. An exclusively African lineage displays an increased number of APL1 paralogs, including the Gambiae species complex and An. christyi (expanded APL1 lineage indicated by shaded box). The five sequenced species of the An. gambiae complex clearly carry three APL1 paralogs, while An. christyi carries more than one and possibly three, but the genome assembly is poor, thus indicated as > 1 APL1 gene. Phylogeny modified from [35] 
christyi genome assembly is fragmented, with an APL1 locus comprised of three unjoined contigs with intervening sequence gaps (Additional file 2: Figure S2).

The next closest sequenced relative to An. christyi, the Asian species An. epiroticus, carries a single APL1 gene (Fig. 1, Additional file 2: Figure S2). Based on synteny and the presence in An. epiroticus of a homolog of the gene AGAP007034 (located between An. gambiae APL1B and APL1C), the single APL1 gene in An. epiroticus displays the greatest relatedness to $A n$. gambiae APL1C, with APL1B and APL1A presumably arising through duplication events during divergence of the Gambiae complex and An. christyi from their common ancestor. The Anopheles species carrying an expanded APL1 gene complement do not correspond precisely to the monophyletic Pyretophorus taxonomic group of Anopheles species [36, 37]. The Pyretophorus group includes An. christyi and the Gambiae complex, which carry an expanded APL1 locus and also An. epiroticus, which has only one APL1 gene. Outside the group of An. christyi and the Gambiae complex, the evidence clearly supports a unique APL1 gene in all species, although resolution in An. minimus is limited due to the poor-quality assembly (Additional file 2: Figure S2). Thus, we conclude that the single APL1 gene found in most sequenced Anopheles including An. stephensi represents the ancestral state of this locus, while the expansion of APL1 to three genes is a derived state, restricted to the Gambiae complex and An. christyi.

Structural comparison of An. stephensi APL1 (628 amino acids) with An. gambiae APL1C, the Gambiae complex basal paralog (730 amino acids), indicates proteins with $50 \%$ amino acid identity and $63 \%$ amino acid similarity. Both are members of the "Long" subfamily of leucine-rich repeat immune (LRIM) proteins [38]. Long subfamily LRIMs contain 10 or more leucinerich repeats. Both An. stephensi APL1 and An. gambiae APL1C contain predicted secretion signal sequences as well as a coiled-coil domain and a characteristic pattern of cysteine residues represented as C - CC - - C, where the single dash represents 10 amino acids and the double dash represents 30 amino acids. The only notable difference is the absence in An. stephensi APL1 of the "PANGGL" domain present in An. gambiae APL1C and some alleles of APL1A, a tandemly repeated peptide sequence with unknown function [25].

\section{APL1 population variation}

Genetic polymorphism within the unique APL1 gene in $A n$. stephensi was measured by sequencing of individual mosquitoes colonized from the natural population in Iran (Additional file 5: Alignment S2). The unique ancestral APL1 gene in these mosquitoes segregates 7 SNP sites over $1190 \mathrm{bp}$, or $\sim 6$ variable nucleotide sites per kilobase (kb). By comparison, the APL1C paralog in the An. coluzzii Ngousso colony from Cameroun, measured in the same way, segregates 117 SNP sites in 2924 $\mathrm{bp}$, or $\sim 40$ variable sites per kb [24], more than six-fold greater polymorphism than the unique An. stephensi APL1 gene. Anopheles stephensi APL1 is compared to An. coluzzii APL1C because APL1C displays the closest orthology to the unique APL1 (Additional file 2: Figure S2). However, in the natural West African population of An. gambiae and An. coluzzii, paralog APL1A is even more polymorphic than APL1C, displaying approximately double the diversity [25]. The differing levels of diversity of the unique APL1 ancestor and the three APL1 paralogs suggest the genes are exposed to distinct natural selection, likely due to functional differences and points to greater evolutionary constraint upon the single ancestral APL1 gene.

Depletion of An. stephensi APL1 reduces mosquito lifespan Depletion of APL1 in An. stephensi by RNAi-mediated silencing (silencing efficiency shown in Additional file 6: Figure S3) led to significantly elevated mosquito mortality as compared to mosquitoes treated with a control dsRNA, dsGFP. The effect was seen regardless of whether APL1 depletion was followed by a sugar meal or blood meal (Fig. 2a, b) and the reduction of mosquito lifespan was even more pronounced when APL1 silencing was followed by a Plasmodium yoeliiinfective blood meal (Fig. 2c). After parasite infection, $\sim 70 \%$ of APL1-depleted mosquitoes died by day 8 postinfection as compared to $\sim 15 \%$ mortality in the dsGFPtreated controls.

\section{Elevated mortality of APL1-depleted An. stephensi is rescued by antibiotic treatment}

The observed mortality after depletion of an immune gene suggested a potential role in protection from bacterial pathology for APL1 in An. stephensi. The three APL1 paralogs found in the Gambiae complex are known to mediate protection from Plasmodium infection [17], but their involvement in protection against other pathogens including bacteria has not been reported.

To test the hypothesis that An. stephensi APL1 protects from pathogenic bacterial effects, newly emerged adult An. stephensi mosquitoes were fed antibiotics in the sugar meal, were then treated with dsAPL1 or dsGFP and were infected with $P$. yoelii parasites. Antibiotic feeding abolished the elevated mortality associated with loss of APL1 function, even in the most pronounced case of Plasmodium infection (Fig. 2d). The simplest interpretation is that the activity of APL1 is essential to protect $A n$. stephensi from unknown lethal bacterial effects under a range of biological conditions. 

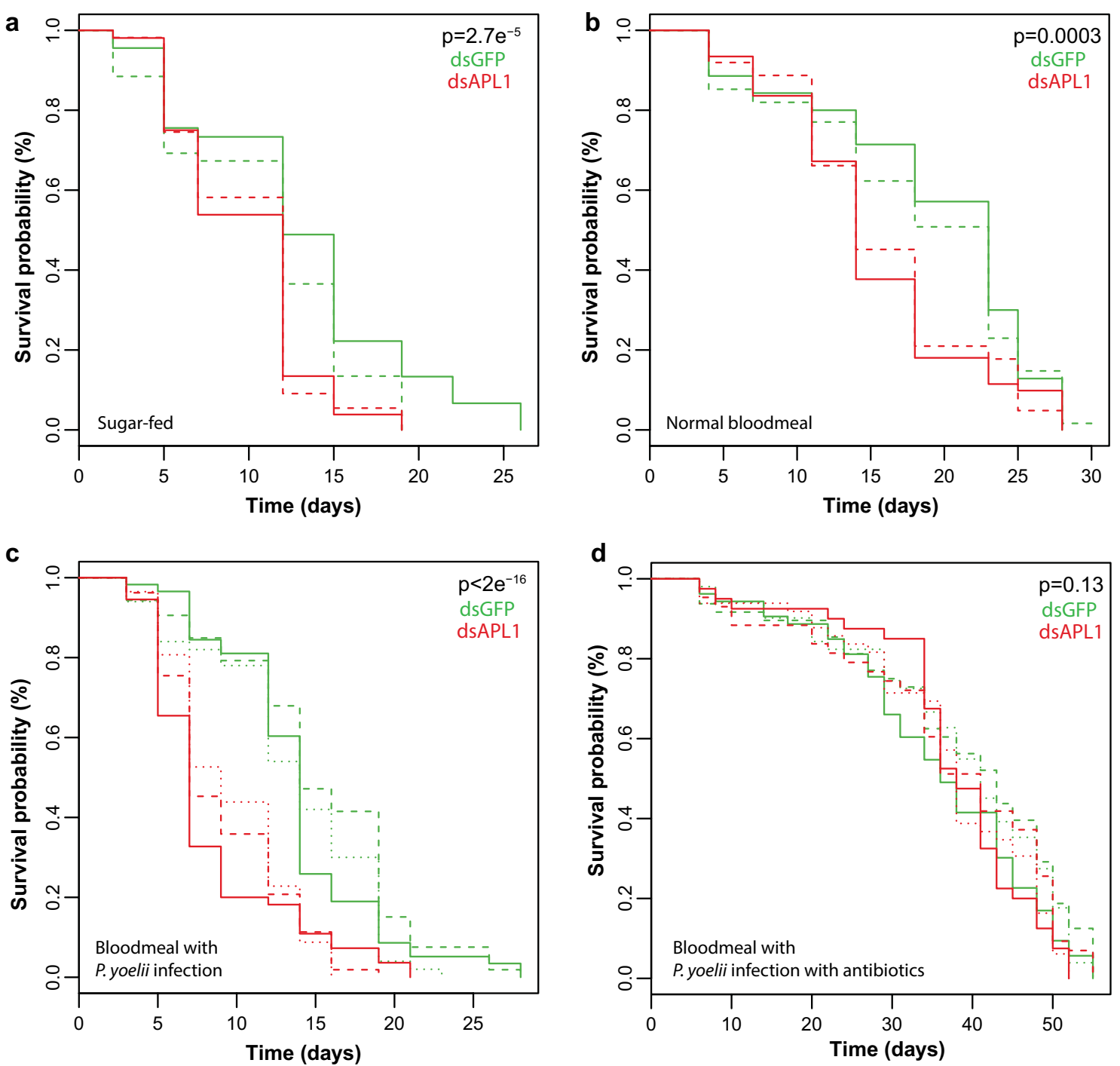

Fig. 2 Depletion of APL1 leads to mosquito mortality in Anopheles stephensi. Survival curves of An. stephensi depleted for APL1 activity by dsAPL1 treatment (red lines) as compared to dsGFP-treated controls (green lines) under different experimental conditions. a Sugar-fed mosquitoes. b Mosquitoes fed an uninfected normal blood meal. c Mosquitoes fed a Plasmodium yoelii-infected blood meal. $\mathbf{d}$ Mosquitoes treated with antibiotics and fed a P. yoelii-infected blood meal. Replicate experiments are distinguished by line type (plain, dashed or dotted, respectively). X-axis indicates time after the start of recording. A Cox proportional hazards regression model was fitted to the data using treatment and replicate as predictor terms. The $P$-value associated with the dsRNA treatment term of the Cox model is shown on each panel. Panel a Wald statistic $=4.195, d f=1, P=$ $2.75 \mathrm{e}^{-5}$; Panel $\mathbf{b}$ Wald statistic $=3.648, d f=1, P=0.0003 ;$ Panel $\mathbf{c}$ Wald statistic $=8.376, d f=1, P<2 \mathrm{e}^{-16} ;$ Panel $\mathbf{d}$ Wald statistic $=1.1518, d f=1, P$ $=0.129$

\section{Simultaneous depletion of all three APL1 paralogs does not reduce An. coluzzii lifespan}

In contrast to the elevated mortality observed in APL1depleted An. stephensi, a mortality effect has not been reported for the APL1 paralogs in An. gambiae and An. coluzzii [11, 14, 16-18]. To confirm this apparent phenotypic difference between the ancestral and expanded APL1 genes, we tested the effect of loss of all APL1 activity in An. coluzzii by depleting all three
APL1 paralogs (silencing efficiency shown in Additional file 6: Figure S3). Simultaneous depletion of all three APL1 paralogs did not alter longevity of An. coluzzii after sugar feeding (Fig. 3a) nor after Plasmodium infection (Fig. 3b). Thus, different from depletion of the single APL1 gene in An. stephensi, which caused elevated mortality under these conditions, activity of the three APL1 paralogs in An. coluzzii do not display the same function. 

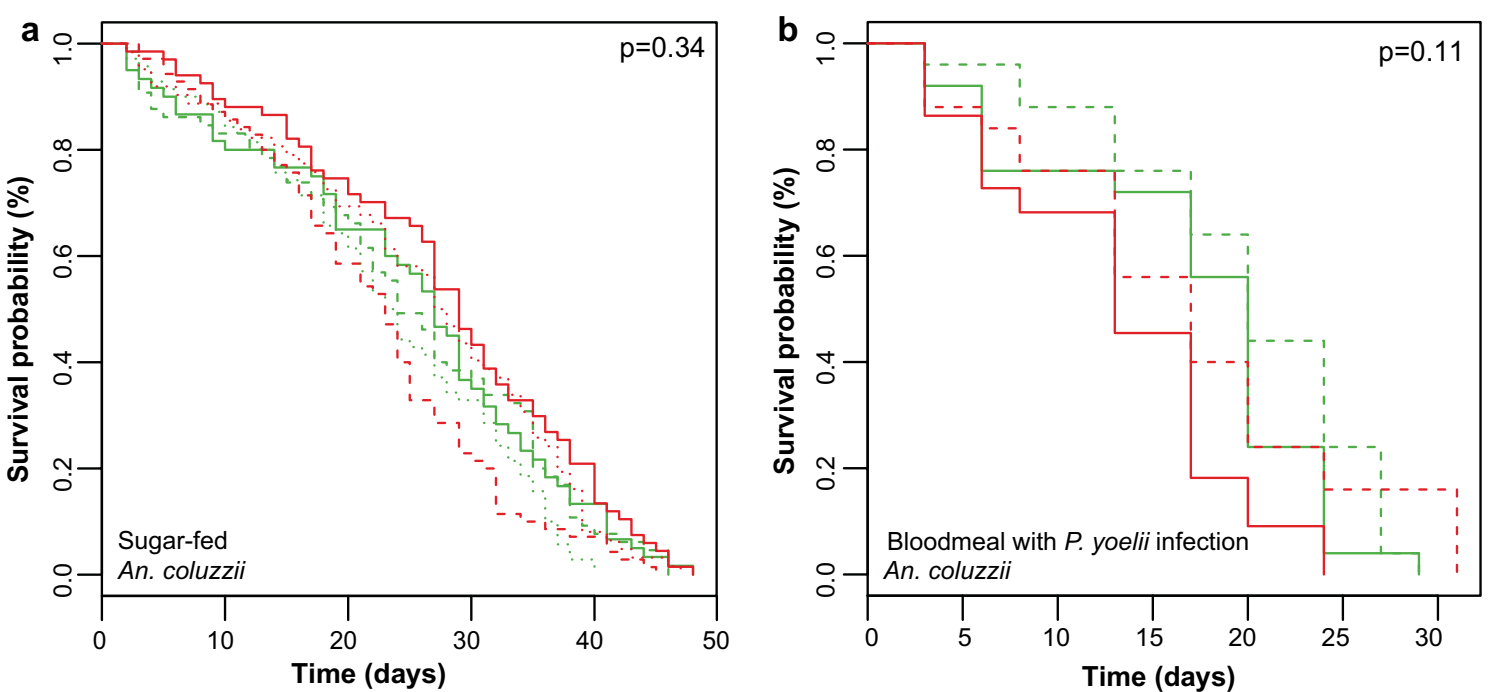

Fig. 3 Simultaneous depletion of all three APL1 paralogs in Anopheles coluzzii does not cause mosquito morality. a Survival curves of An. coluzzii depleted for APL1 activity by dsAPL1 treatment (red lines) as compared to dsGFP controls (green lines), for sugar-fed mosquitoes. b Survival curves for mosquitoes fed a Plasmodium yoelii-infected blood meal. Survival curves from replicates are distinguished by line type (plain, dashed or dotted, respectively). X-axis indicates time after the start of recording, not mosquito age (see Methods). A Cox proportional hazards regression model was fitted to the data using treatment and replicate as predictor terms. The $P$-value associated with the dsRNA treatment term of the Cox model is shown on each panel. Panel a Wald statistic $0.95, d f=1, P=0.34$; Panel b Wald statistic $=1.589, d f=1, P=0.112$
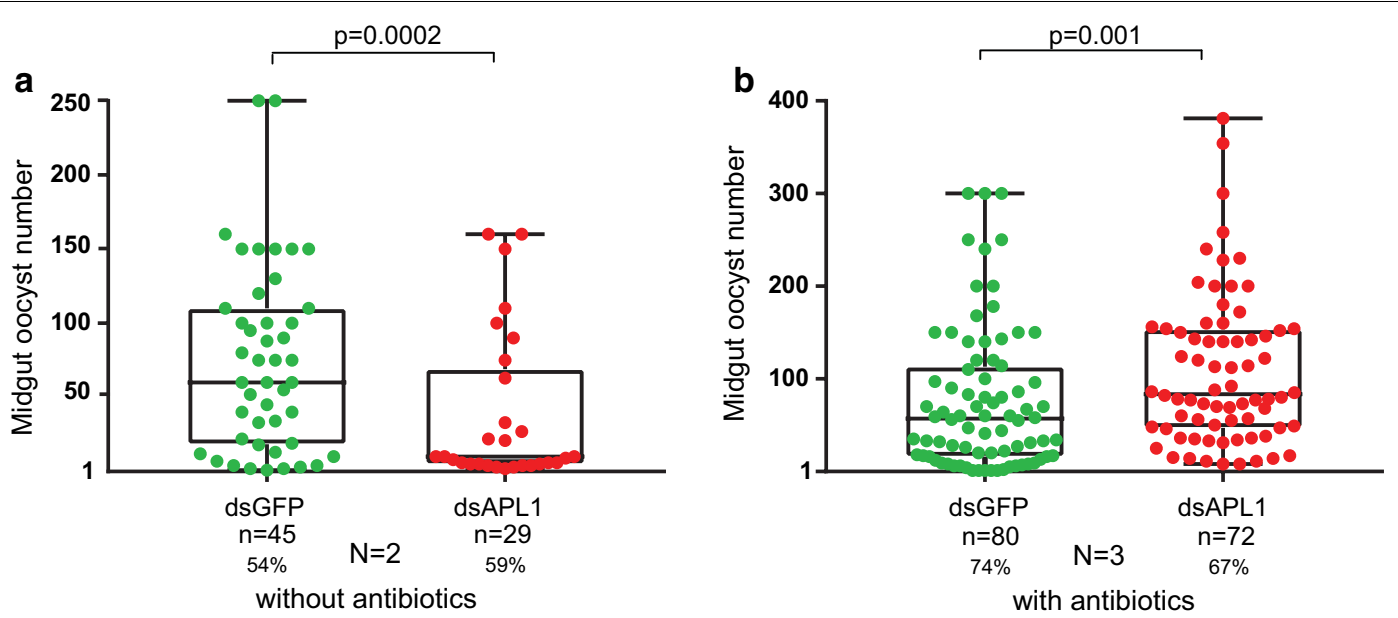

Fig. 4 Anopheles stephensi APL1 protection from Plasmodium yoelii infection is secondary to its antibacterial function. a P. yoelii oocyst infection intensity in An. stephensi mosquitoes treated with dsAPL1 or control dsGFP, both without antibiotic treatment. Oocyst intensity is the oocyst count in mosquitoes with $\geq 1$ oocyst, to avoid confounding with infection prevalence. Oocyst infection prevalence, the proportion of mosquitoes carrying $\geq 1$ oocyst, is indicated as a percentage below sample sizes. Number of biological replicates is indicated below plots. Combined $P$-value: $X^{2}=22.3529, d f=4, P=-0.0002$ (Replicate $1, W=30.5, P=0.0075$; Replicate $2, W=226.5, P=0.002$ ). $\mathbf{b}$ As in $\mathbf{a}$, but mosquitoes were subject to antibiotic treatment before Plasmodium exposure. Combined P-value, $X^{2}=21.85, d f=6, P=0.001$ (Replicate 1, W $=1144.5, P=0.009$; Replicate 2, $W=463.5, P=0.043 ;$ Replicate $3, W=40, P=0.05549)$

\section{Anti-Plasmodium protection by An. stephensi APL1}

\section{is secondary to protection from elevated mortality}

Depletion of the unique APL1 gene in An. stephensi led to decreased $P$. yoelii parasite load (Fig. 4a). However, the APL1-depleted An. stephensi were already compromised due to their elevated mortality and we hypothesized that they may have been physiologically unable to support Plasmodium development. In the presence of antibiotics, these mosquitoes carried significantly greater $P$. yoelii infection loads as compared to dsGFP-treated controls 

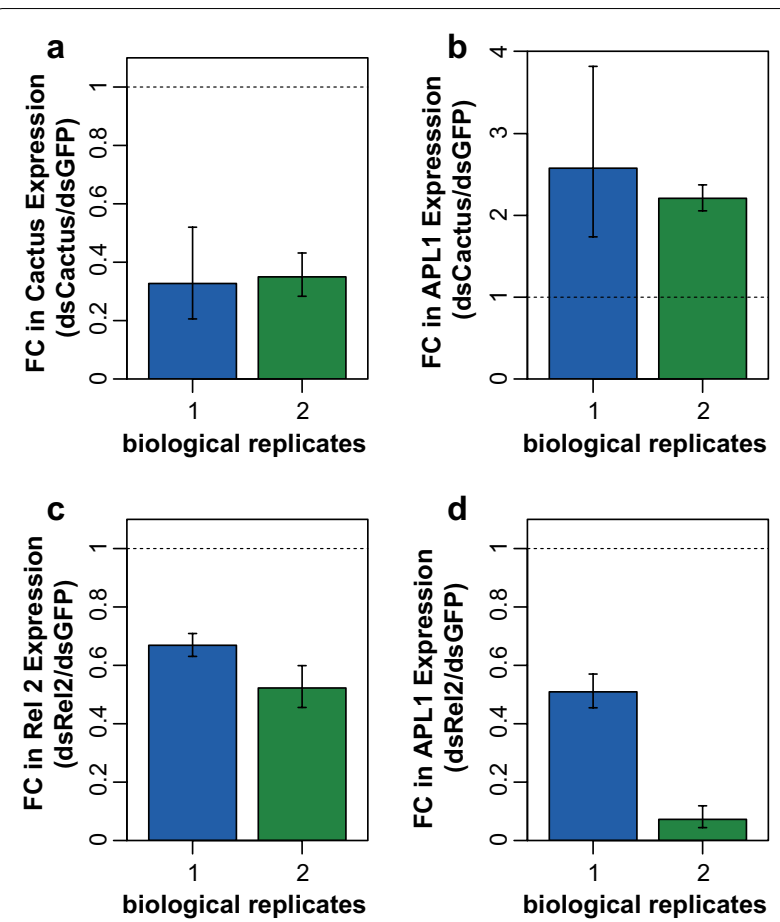

Fig. 5 Transcription of Anopheles stephensi APL1 is regulated by both Toll and Imd immune signaling pathways. Regulation of expression of the unique An. stephensi APL1 gene was queried by silencing the negative regulator of Toll, Cactus ( $\mathbf{a}$ and $\mathbf{b}$ ) or the positive regulator of Imd, Rel2 (c and $\mathbf{d}$ ). a Cactus expression is efficiently repressed by treatment with dsRNA targeting Cactus (dsCactus). Graph indicates fold change of Cactus expression by dsCactus treatment as compared to dsGFP controls. $\mathbf{b}$ APL1 expression is augmented by silencing of Cactus, which constitutively activates the Toll pathway. Graph indicates fold change of APL1 gene expression in An. stephensi depleted for Cactus by dsCactus treatment, relative to dsGFP treated controls. c Rel2 expression is efficiently suppressed by treatment with dsRNA targeting Rel2 (dsRel2). Graph indicates fold change of Rel2 expression by dsRel 2 treatment as compared to dsGFP controls. d APL1 expression is diminished by silencing of Rel2, which inhibits Imd pathway activity. Graph indicates fold change of APL1 gene expression in An. stephensi depleted for Rel2 by dsRel2 treatment, relative to dsGFP treated controls. Transcript abundance is measured by quantitative RT-PCR in two biological replicates as indicated

(Fig. 4b). Thus, controlling for the mortality effect of APL1 depletion in An. stephensi revealed an underlying anti-Plasmodium activity of the unique APL1 gene, but the dominant function of APL1 appears to be protection from an elevated mortality phenotype that is complemented by antibiotic provision.

These results are in contrast to silencing of the three APL1 paralogs in An. coluzzii, which consistently leads to elevated Plasmodium infection levels $[16,17]$ but not elevated mortality (Fig. 3). Therefore, the three APL1 paralogs confer protection against Plasmodium infection independently of the need to protect against mortality from potential bacterial effects. Taken together, these results indicate that the combined phenotype of the three paralogs does not recapitulate the phenotype of the ancestral single gene and that the divergence of the three APL1 paralogs from the unique APL1 ancestor was accompanied by important functional changes. Protection from pathogenic bacterial effects may have been functionally replaced in the expanded-APL1 mosquito lineage by other unknown immune factors or distinct physiological mechanisms.

\section{APL1 in An. stephensi is regulated by both the Toll and Imd signaling pathways}

The APL1 paralogs in An. coluzzii are transcriptionally regulated by distinct immune signaling pathways. Expression of paralog APL1A is regulated by the transcription factor Rel2, the positive regulator of the Immune deficiency (Imd) immune pathway, while paralog APL1C is regulated by transcription factor Rel1, positive regulator of the Toll pathway $[14,16,17,39]$.

We tested the effect of these signaling pathways on expression of the unique APL1 gene in An. stephensi. Activation of Toll signaling in An. stephensi by depletion of the Toll negative regulator, Cactus (Fig. 5a), led to increased APL1 expression (Fig. 5b) and depletion of the Imd positive regulator Rel2 (Fig. 5c) led to reduced APL1 expression (Fig. 5d). Consequently, An. stephensi APL1 expression is under control of both the Toll and Imd pathways. A previous study found that overexpression of a Rel2 transgene in An. stephensi induced APL1 expression, consistent with our findings, but the response to Rel1 was not tested [20]. Thus, the ancestral unique APL1 gene in $A n$. stephensi is regulated by two signaling pathways, Toll and Imd, while after APL1 gene duplication and divergence, these two controlling pathways were subdivided to control of the derived paralogs APL1C and APL1A, respectively.

\section{Discussion}

We find that An. stephensi and most other sequenced Anopheles species carry a single APL1 gene, which expanded to a family of three paralogs in an exclusively African lineage that includes all members of the Gambiae complex and $A n$. christyi. Silencing of the unique ancestral APL1 gene in An. stephensi led to elevated mosquito mortality, reversed by antibiotic treatment, which suggested a role for APL1 in protection from pathogenic bacterial effects. The highest mortality was detected in APL1-depleted An. stephensi mosquitoes after Plasmodium infection, as compared to after a sugar meal or normal blood meal. This result suggests that enteric bacteria could underlie the mortality seen in APL1-depleted $A n$. stephensi, because malaria ookinete invasion from the midgut lumen facilitates physical entry of bacteria into epithelial cells and heightens microbial exposure [40]. 
Thus, we infer that activity of the unique APL1 gene protects An. stephensi from effects of the enteric microbiome that are lethal in the absence of APL1. Further work will be required to determine the mechanisms of the bacterial effect and APL1 protection. APL1 could function to modulate bacterial abundance, controlling either specific bacterial taxa or protecting from general dysbiosis; or could mediate tolerance to the stress of bacterial effects such as virulence factors or toxins. APL1 effects on bacteria could potentially influence blood digestion or the peritrophic matrix, although this could not be a primary explanation because elevated mortality after depletion of APL1 is observed with or without a blood meal. Taking into account the present results and the biology of APL1, which is known to be at least a soluble hemolymph factor in An. gambiae, we speculate that the unique APL1 in An. stephensi may function to protect the hemocoel compartment from enteric bacteria, either as a hemocoel barrier against bacterial escape from the midgut, or as a tolerance factor buffering bacterial pathology.

The function of the unique APL1 gene is distinct from that of the expanded APL1 gene family in An. coluzzii, which protects against Plasmodium but is not essential for protection from bacterial effects. The unique APL1 gene displays an ancestral immune signaling profile, because its expression is regulated by both Toll and Imd pathways, in distinction to the paralogs in An. coluzzii, in which regulation by the immune pathways specialized to different gene family members.

\section{Function of ancestral and derived APL1 genes}

The unique APL1 gene is essential for An. stephensi fitness and survival, while the three paralogs combined are not essential for An. coluzzii under the same conditions, because their depletion does not have lethal consequences. Gene essentiality is dependent on genomic and biological context, including environmental conditions [41]. The common ancestor of the Gambiae complex-An. christyi lineage evolved to exploit an unknown ecological niche, probably in Africa because all species known to carry an expanded APL1 locus are African, and may have encountered new environmental pathogens there [25, 42, 43]. It appears that essentiality of the ancestral unique APL1 gene was lost at the time of the expansion and functional divergence of the three paralogs. The expanded APL1 paralogs evolved new immune roles, exemplified by observed functional differences among the three paralogs in the Gambiae complex [1618]. However, the paralogs did not simply subdivide the functions of the unique ancestor, because they are not required for protection against bacterial effects under the conditions tested. The expansion of the APL1 gene family was likely accompanied by a suite of other unknown genomic changes necessary for adaptation of the Gambiae complex-An. christyi lineage to the new ecological niche, potentially in other immune factors that interact with APL1 such as TEP1 and LRIM1, but this remains to be described. Protection of Anopheles from these pathogenic bacterial effects was presumably shifted to other unknown genes or physiological factors, which may have evolved at the same time.

Previous population sequencing revealed that the three APL1 paralogs in the Gambiae complex are exceptionally polymorphic and display signals of adaptive maintenance of variation, including maintenance of alleles that are older than the species of the Gambiae complex [25]. This genetic pattern is consistent with a model of balancing polymorphism maintained by exposure to fluctuating environmental pathogens in a trench warfare dynamic [44]. In contrast, examination of An. stephensi APL1 sequences from individual mosquitoes from the Iran population suggests that diversity of the unique APL1 gene is quite low. One potential explanation could be that unique APL1 is under selection mainly to protect the host from relatively stable taxa of enteric bacteria, which could be commensals of the microbiome. Additional population re-sequencing will be required to test these hypotheses.

\section{APL1 copy number and malaria vectorial capacity}

Expanded APL1 copy number does not directly correlate with dominant vector species (DVS) status, but this comparison is confounded with mosquito behavior, because not all expanded-APL1 species are human feeding. The four expanded-APL1 species that are DVS display high human-biting preference (An. gambiae, An. coluzzii, An. merus, An. melas), while the other two sequenced species with an expanded APL1 locus, the non-vectors An. christyi and An. quadriannulatus, are cattle-feeding species in nature $[45,46]$. Of these latter two non-vector species, An. quadriannulatus is physiologically susceptible to infection with $P$. falciparum when fed on parasitemic blood [46, 47] and permissiveness of An. christyi to infection has not been tested.

The more interesting question is whether, among human-feeding species, carriage of the expanded APL1 locus influences the efficiency of malaria transmission. The human-feeding members of the Gambiae complex are considered the most efficient malaria vectors in the world $[48,49]$ and all of these species carry the expanded APL1 locus. Their efficient malaria transmission could be a secondary consequence of inhabiting African ecological niches that also happen to be particularly favorable to malaria transmission $[7,8,10]$. However, other African vectors such as An. funestus, An. nili, An. pharoensis and An. moucheti are DVS, but are often described as 
locally important secondary vectors and lack the epidemiological impact of the expanded-APL1 Gambiae complex. Anopheles funestus carries a single APL1 gene and An. nili, An. pharoensis and An. moucheti have not been sequenced but based on the phylogenetic analysis are also expected to carry the single ancestral APL1 locus.

Thus, the present results raise the question whether the observed correlation of APL1 copy number (and other unknown associated genomic changes) with vectorial efficiency is accidental or biologically meaningful. The ancestral single APL1 protects An. stephensi against malaria parasite infection, but this activity is secondary to a dominant and essential function of protection against bacterial effects. Under these conditions, it would not seem adaptive for Plasmodium to inhibit the activity of the unique APL1 in order to modulate anti-malaria immunity, because parasite inhibition of unique APL1 immune function in An. stephensi would be expected to decrease vector survival and therefore the parasite's own reproductive fitness. In contrast, in An. coluzzii with three APL1 paralogs, malaria immunity and protection from bacterial effects are uncoupled, because loss of APL1 function does not reduce longevity. The separation of anti-Plasmodium immunity and protection from bacterial pathology should allow Plasmodium (and other pathogens) to subvert APL1mediated immunity without the risk of provoking host mortality.

\section{Conclusions}

The ancestral and derived APL1 loci, represented by $A n$. stephensi and An. coluzzii, respectively, display large differences in gene essentiality, function, regulation and genetic diversity. Manipulative experimentation and population genetic analysis will be required to understand the functional and ecological significance of the ancestral and derived APL1 for immunity and malaria transmission in An. stephensi and other species.

\section{Supplementary information}

Supplementary information accompanies this paper at https://doi. org/10.1186/s13071-019-3868-y.

Additional file 1: Figure S1. Manual sequence confirmation of unique APL1 gene in Anopheles stephensi strain SDA-500. Detailed legend in figure file.

Additional file 2: Figure S2. APL1 gene copy number in Anopheles genome sequence assemblies. Detailed legend in figure file.

Additional file 3: Table S1. Primer sequences.

Additional file 4: Alignment S1. FASTA-format sequence data file for manual validation of single APL1 gene in Anopheles stephensi. By chromosome synteny, the Anopheles stephensi intergenic region between the APL1 gene (ASTE016290 in the SDA-500 assembly) and the adjacent gene (ASTE008334) would contain other APL1 family paralogs, if other APL1 genes exist in An. stephensi. The $\sim 7 \mathrm{~kb}$ intergenic region contains unjoined sequences in the SDA-500 assembly and thus the true size was unknown. The region was amplified and Sanger sequenced by the strategy indicated in Additional file 1: Figure S1 and amplicons were assembled as a single contig, resolving the region between primer ST11 and ST25 (Additional file 3: Table S1). The resolved sequence does not contain sequences related to APL1, confirming the absence of APL1 paralogs in An. stephensi.

Additional file 5: Alignment S2. FASTA-format sequence data file of amplicon sequences for population variation of APL1 gene in Anopheles stephensi. Amplicons of the APL1 gene in Anopheles stephensi were amplified from wild mosquitoes and colonies collected in Iran using primers Iran40F and Iran06R and Sanger sequenced.

Additional file 6: Figure S3. Efficiency of APL1 gene silencing. Detailed legend in figure file.

\section{Abbreviations}

bp: base pairs; d: days; dsRNA: double strand RNA; DVS: dominant malaria vector species; RNAi: RNA interference.

\section{Acknowledgements}

We thank the Institut Pasteur core facility, the Center for the Production and Infection of Anopheles (CEPIA), for gametocyte culture and mosquito infection and Corinne Genève, GGIV Institut Pasteur, for rearing and infecting mosquitoes. We thank Olivier Sylvie, Inserm unit on Molecular biology and immunology of malaria liver infection, for provision of P. yoelii fluorescent strain delta-p230p-GFP.

\section{Authors' contributions}

Designed research: CM, EB, KE, NDD, NS, MMR and KDV. Performed research: $C M, E B, K E, I H, C D, E B F, A R, S Z, M I K N$, NDD and MMR. Analyzed data: CM, EB, KE, $I H, C D, A R, S Z, M I K N, M M R$ and KDV. Wrote the paper: CM, EB, KE, IH, NDD, MMR and KDV. All authors read and approved the final manuscript.

\section{Funding}

This study received financial support to KDV from the European Commission, Horizon 2020 Infrastructures \#731060 Infravec2; European Research Council, Support for frontier research, Advanced Grant \#323173 AnoPath; Agence Nationale de la Recherche, \#ANR-19-CE35-0004 ArboVec; and French Laboratoire d'Excellence "Integrative Biology of Emerging Infectious Diseases" \#ANR-10-LABX-62-IBEID and to MMR from National Institutes of Health, NIAID \#Al121587. Funders had no role in study design, data collection and analysis, decision to publish, or preparation of the manuscript.

\section{Availability of data and materials}

All newly generated sequences are available within the present article as Additional file 4: Alignment S1 and Additional file 5: Alignment S2.

\section{Ethics approval and consent to participate}

The protocol for the ethical treatment of the animals used in this study was approved by the research animal ethics committee of the Institut Pasteur, "C2EA-89 CETEA Institut Pasteur" as protocol number B75-15-31. The Institut Pasteur ethics committee is authorized by the French Ministry of Higher Education and Research (MESR) under French law N ${ }^{\circ} 2001-486$, which is aligned with Directive 2010/63/EU of the European Commission on the protection of animals used for scientific purposes.

\section{Consent for publication}

Not applicable.

\section{Competing interests}

The authors declare that they have no competing interests.

\section{Author details}

${ }^{1}$ Unit of Insect Vector Genetics and Genomics, Department of Parasites and Insect Vectors, Institut Pasteur, Paris, France. ${ }^{2}$ CNRS Unit of Evolutionary Genomics, Modeling and Health (UMR2000), Institut Pasteur, Paris, France. ${ }^{3}$ Present Address: Wadsworth Center, New York State Department of Health, Slingerlands, NY, USA. ${ }^{4}$ Malaria and Vector Research Group, Biotechnology 
Research Center, Institut Pasteur of Iran, Tehran, Iran. ${ }^{5}$ Department of Microbiology and Immunology, Medical College of Wisconsin, Milwaukee, WI, USA.

\section{Received: 28 September 2019 Accepted: 19 December 2019}

\section{Published online: 13 January 2020}

\section{References}

1. Sinka ME, Bangs MJ, Manguin S, Rubio-Palis Y, Chareonviriyaphap T, Coetzee $\mathrm{M}$, et al. A global map of dominant malaria vectors. Parasit Vectors. 2012;5:69.

2. Seyfarth M, Khaireh BA, Abdi AA, Bouh SM, Faulde MK. Five years following first detection of Anopheles stephensi (Diptera: Culicidae) in Djibouti, Horn of Africa: populations established-malaria emerging. Parasitol Res. 2019;118:725-32.

3. Surendran SN, Sivabalakrishnan K, Gajapathy K, Arthiyan S, Jayadas TTP, Karvannan K, et al. Genotype and biotype of invasive Anopheles stephensi in Mannar Island of Sri Lanka. Parasit Vectors. 2018;11:3.

4. Service MW. Mosquito ecology: field sampling methods. 2nd ed. London: Chapman \& Hall; 1993.

5. Cohuet A, Harris C, Robert V, Fontenille D. Evolutionary forces on Anopheles: what makes a malaria vector? Trends Parasitol. 2010;26:130-6.

6. Gething PW, Patil AP, Smith DL, Guerra CA, Elyazar IR, Johnston GL, et al. A new world malaria map: Plasmodium falciparum endemicity in 2010. Malar J. 2011;10:378.

7. Weiss DJ, Mappin B, Dalrymple U, Bhatt S, Cameron E, Hay SI, et al. Reexamining environmental correlates of Plasmodium falciparum malaria endemicity: a data-intensive variable selection approach. Malar J. 2015;14:68

8. Bhatt S, Weiss DJ, Cameron E, Bisanzio D, Mappin B, Dalrymple U, et al. The effect of malaria control on Plasmodium falciparum in Africa between 2000 and 2015. Nature. 2015:526:207-11.

9. Coulibaly B, Kone R, Barry MS, Emerson B, Coulibaly MB, Niare O, et al. Malaria vector populations across ecological zones in Guinea Conakry and Mali, West Africa. Malar J. 2016;15:191.

10. Riehle MM, Bukhari T, Gneme A, Guelbeogo WM, Coulibaly B, Fofana $\mathrm{A}$, et al. The Anopheles gambiae 2La chromosome inversion is associated with susceptibility to Plasmodium falciparum in Africa. Elife. 2017:6:e25813.

11. Riehle MM, Markianos $\mathrm{K}$, Niaré $\mathrm{O}, \mathrm{Xu} J, \mathrm{Li}$ J, Touré AM, et al. Natural malaria infection in Anopheles gambiae is regulated by a single genomic control region. Science. 2006;312:577-9.

12. Mitri C, Markianos K, Guelbeogo WM, Bischoff E, Gneme A, Eiglmeier K, et al. The kdr-bearing haplotype and susceptibility to Plasmodium falciparum in Anopheles gambiae: genetic correlation and functional testing. Malar J. 2015;14:391.

13. Povelones M, Waterhouse RM, Kafatos FC, Christophides GK. Leucine-rich repeat protein complex activates mosquito complement in defense against Plasmodium parasites. Science. 2009:324:258-61.

14. Riehle MM, Xu J, Lazzaro BP, Rottschaefer SM, Coulibaly B, Sacko M, et al. Anopheles gambiae APL1 is a family of variable LRR proteins required for Rel1-mediated protection from the malaria parasite, Plasmodium berghei. PLOS ONE. 2008;3:e3672.

15. Blandin S, Shiao SH, Moita LF, Janse CJ, Waters AP, Kafatos FC, et al. Complement-like protein TEP1 is a determinant of vectorial capacity in the malaria vector Anopheles gambiae. Cell. 2004:116:661-70.

16. Mitri C, Jacques JC, Thiery I, Riehle MM, Xu J, Bischoff E, et al. Fine pathogen discrimination within the APL1 gene family protects Anopheles gambiae against human and rodent malaria species. PLoS Pathog. 2009:5:e1000576.

17. Mitri C, Bischoff E, Takashima E, Williams M, Eiglmeier K, Pain A, et al. An evolution-based screen for genetic differentiation between Anopheles sister taxa enriches for detection of functional immune factors. PLoS Pathog. 2015;11:e1005306.

18. Garver LS, Bahia AC, Das S, Souza-Neto JA, Shiao J, Dong Y, et al. Anopheles Imd pathway factors and effectors in infection intensity-dependent anti-Plasmodium action. PLoS Pathog. 2012;8:e1002737.
19. Jiang X, Peery A, Hall AB, Sharma A, Chen XG, Waterhouse RM, et al. Genome analysis of a major urban malaria vector mosquito, Anopheles stephensi. Genome Biol. 2014;15:459.

20. Dong Y, Das S, Cirimotich C, Souza-Neto JA, McLean KJ, Dimopoulos G. Engineered anopheles immunity to Plasmodium infection. PLoS Pathog. 2011;7:e1002458.

21. Souvannaseng L, Hun LV, Baker H, Klyver JM, Wang B, Pakpour N, et al. Inhibition of JNK signaling in the Asian malaria vector Anopheles stephensi extends mosquito longevity and improves resistance to Plasmodium falciparum infection. PLoS Pathog. 2018;14:e1007418.

22. Feldmann AM, Ponnudurai T. Selection of Anopheles stephensi for refractoriness and susceptibility to Plasmodium falciparum. Med Vet Entomol. 1989:3:41-52.

23. Harris C, Lambrechts L, Rousset F, Abate L, Nsango SE, Fontenille D, et al. Polymorphisms in Anopheles gambiae immune genes associated with natural resistance to Plasmodium falciparum. PLoS Pathog. 2010;6:e1001112.

24. Holm I, Lavazec C, Garnier T, Mitri C, Riehle MM, BischoffE, et al. Diverged alleles of the Anopheles gambiae leucine-rich repeat gene APL1A display distinct protective profiles against Plasmodium falciparum. PLOS ONE. 2012; 7:e52684.

25. Rottschaefer SM, Riehle MM, Coulibaly B, Sacko M, Niaré O, Morlais I, et al. Exceptional diversity, maintenance of polymorphism, and recent directional selection on the APL1 malaria resistance genes of Anopheles gambiae. PLoS Biol. 2011;9:e1000600.

26. Giraldo-Calderón Gl, Emrich SJ, MacCallum RM, Maslen G, Dialynas E, Topalis $P$, et al. VectorBase: an updated bioinformatics resource for invertebrate vectors and other organisms related with human diseases. Nucleic Acids Res. 2015;43(Database issue):D707-13.

27. Carver TJ, Rutherford KM, Berriman M, Rajandream MA, Barrell BG, Parkhill J. ACT: the artemis comparison tool. Bioinformatics. 2005;21:3422-3.

28. Sullivan MJ, Petty NK, Beatson SA. Easyfig: a genome comparison visualizer. Bioinformatics. 2011;27:1009-10.

29. Mitchell AL, Attwood TK, Babbitt PC, Blum M, Bork P, Bridge A, et al. InterPro in 2019: improving coverage, classification and access to protein sequence annotations. Nucleic Acids Res. 2019;47(D1):D351-60.

30. Manzoni G, Briquet S, Risco-Castillo V, Gaultier C, Topcu S, Ivanescu ML, et al. A rapid and robust selection procedure for generating drug-selectable marker-free recombinant malaria parasites. Sci Rep. 2014;4:4760.

31. Fisher RA. Statistical methods for research workers. Edinburgh: Oliver \& Boyd; 1925.

32. R Core Team. R: A language and environment for statistical computing. Vienna, Austria: R Foundation for Statistical Computing; 2012. http:// www.R-project.org/.

33. Cox DR. Regression models and life-tables. J R Stat Soc Series B Stat Methodol. 1972:34:187-220.

34. Grambsch PM, Therneau TM. Proportional hazards tests and diagnostics based on weighted residuals. Biometrika. 1994;81:515-26.

35. Neafsey DE, Waterhouse RM, Abai MR, Aganezov SS, Alekseyev MA, Allen JE, et al. Mosquito genomics. Highly evolvable malaria vectors: the genomes of 16 Anopheles mosquitoes. Science. 2015;347:1258522.

36. Hao YJ, Zou YL, Ding YR, Xu WY, Yan ZT, Li XD, et al. Complete mitochondrial genomes of Anopheles stephensi and An. dirus and comparative evolutionary mitochondriomics of 50 mosquitoes. Sci Rep. 2017;7:7666.

37. Harbach RE, Kitching IJ. The phylogeny of Anophelinae revisited: inferences about the origin and classification of Anopheles (Diptera: Culicidae). Zool Scr. 2016:45:34-47.

38. Waterhouse RM, Povelones M, Christophides GK. Sequence-structurefunction relations of the mosquito leucine-rich repeat immune proteins. BMC Genomics. 2010;11:531.

39. Frolet C, Thoma M, Blandin S, Hoffmann JA, Levashina EA. Boosting NF-kappaB-dependent basal immunity of Anopheles gambiae aborts development of Plasmodium berghei. Immunity. 2006;25:677-85.

40. Seitz HM, Maier WA, Rottok M, Becker-Feldmann H. Concomitant infections of Anopheles stephensi with Plasmodium berghei and Serratia marcescens: additive detrimental effects. Zentralbl Bakteriol Mikrobiol Hyg A. 1987:266:155-66.

41. Rancati G, Moffat J, Typas A, Pavelka N. Emerging and evolving concepts in gene essentiality. Nat Rev Genet. 2018;19:34-49. 
42. Mitri C, Vernick KD. Anopheles gambiae pathogen susceptibility: the intersection of genetics, immunity and ecology. Curr Opin Microbiol. 2012;15:285-91.

43. White BJ, Lawniczak MK, Cheng C, Coulibaly MB, Wilson MD, Sagnon $\mathrm{N}$, et al. Adaptive divergence between incipient species of Anopheles gambiae increases resistance to Plasmodium. Proc Natl Acad Sci USA. 2011;108:244-9.

44. Stahl EA, Dwyer G, Mauricio R, Kreitman M, Bergelson J. Dynamics of dis ease resistance polymorphism at the Rpm1 locus of Arabidopsis. Nature. 1999;400:667-71.

45. Animut A, Balkew M, Gebre-Michael T, Lindtjørn B. Blood meal sources and entomological inoculation rates of anophelines along a highland altitudinal transect in south-central Ethiopia. Malar J. 2013;12:76.

46. Takken W, Eling W, Hooghof J, Dekker T, Hunt R, Coetzee M. Susceptibility of Anopheles quadriannulatus Theobald (Diptera: Culicidae) to Plasmodium falciparum. Trans R Soc Trop Med Hyg. 1999;93:578-80.
47. Habtewold T, Povelones M, Blagborough AM, Christophides GK. Transmission blocking immunity in the malaria non-vector mosquito Anopheles quadriannulatus species A. PLoS Pathog. 2008;4:e1000070.

48. The Anopheles gambiae Genomes Consortium. Genetic diversity of the African malaria vector Anopheles gambiae. Nature. 2017;552:96-100.

49. Kiszewski A, Mellinger A, Spielman A, Malaney P, Sachs SE, Sachs J. A global index representing the stability of malaria transmission. Am J Trop Med Hyg. 2004;70:486-98.

\section{Publisher's Note}

Springer Nature remains neutral with regard to jurisdictional claims in published maps and institutional affiliations.
Ready to submit your research? Choose BMC and benefit from:

- fast, convenient online submission

- thorough peer review by experienced researchers in your field

- rapid publication on acceptance

- support for research data, including large and complex data types

- gold Open Access which fosters wider collaboration and increased citations

- maximum visibility for your research: over 100M website views per year

At BMC, research is always in progress.

Learn more biomedcentral.com/submissions 